\title{
Qualified Promise: DNA Methylation Assays for the Detection and Classification of Human Cancers
}

\author{
Wolfgang Schulz \\ Department of Urology, Heinrich Heine University, Moorenstrasse 5, Duesseldorf 40225, Germany
}

In the last decade, epigenetic changes have been recognized as important factors in the development and progression of human cancers. Specifically, alterations of DNA methylation patterns are now thought to contribute to altered gene expression and chromosomal instability, to an often comparable degree as genetic alterations such as point mutations, insertions, deletions, chromosomal translocations, gains, and losses.

Of note, DNA methylation patterns in cancer cells can change in both directions, that is, decreases as well as increases are found. Decreased methylation ("hypomethylation") and increased methylation ("hypermethylation") can occur independently of each other, but also simultaneously. While some methylation changes are subtle, two stand out as clearly pathological. The sequences surrounding the transcriptional start sites of many genes are particularly rich in the dinucleotide $\mathrm{CpG}$ compared to the rest of the genome. Many of these sequences, termed "CpG-islands," are normally devoid of DNA methylation. Therefore, hypermethylation of such CpG-islands is evidently an aberrant process that is largely restricted to cancers. Limited and "patchy" CpG-island hypermethylation is observed in preneoplastic and aging tissues, while in some cancers, hundreds of CpG-islands become densely hypermethylated. Conversely, some sequences that are densely methylated in somatic cells become hypomethylated. In normal somatic cells, the CpG-rich satellite sequences SAT2 and SAT3 and interspersed CpG-rich retrotransposons like LINE-1 and ALU are strongly methylated. As these repeat sequences constitute the bulk of

Correspondence and reprint requests to Wolfgang Schulz, Department of Urology, Heinrich Heine University, Moorenstrasse 5, Duesseldorf 40225, Germany, E-mail: wolfgang.schulz@uniduesseldorf.de

This is an open access article distributed under the Creative Commons Attribution License which permits unrestricted use, distribution, and reproduction in any medium, provided the original work is properly cited. the genome and become hypomethylated in many cancers, hypomethylation is typically the dominant alteration. Methylation of repeat sequences decreases in many cancers in a seemingly unspecific fashion, but hypomethylation also affects a number of single-copy genes. Importantly, neither hypomethylation of single-copy genes nor hypermethylation of CpG-islands seem to occur in a random manner and show considerable differences between cancer types and subtypes. The hypermethylation of a CpG-island or the hypomethylation of a gene promoter that is methylated in normal somatic tissue therefore strongly hints at a functional importance in each individual case. Accordingly, hypermethylation of CpGisland promoters is a common mechanism for inactivation of tumor suppressor genes in cancer and hypomethylation of certain genes may be required to equip tumor cells with proteins necessary for invasion and metastasis. On a note of caution, though, it is rather unlikely that every instance of altered gene methylation implies important functional significances. For instance, tumor cells may benefit from "slimming down" gene expression by hypermethylation of "unnecessary" genes, but their growth may not depend on this change.

Apart from its functional significance, altered DNA methylation in cancer has attracted great attention as a tool for cancer diagnosis. A large variety of techniques are now available to determine DNA methylation of the genome at large, of specific sites in specific genes, of specific regions, or of multiple genes. They comprise "classical" blotting techniques relying on methylationsensitive restriction enzymes, a host of PCR techniques, usually following bisulfite treatment of DNA which converts only unmethylated cytosines to uracil, HPLC-based methodology, several variants of mass spectometry, affinity chromatography using, for example, methylcytosine binding proteins, and various array techniques. Two obvious trends in the application of methylation methodology are an emphasis on improved quantification, exemplified by the use of quantitative (real-time) PCR techniques, and determination of methylation at multiple genes, using 
multiplex PCR, arrays, or mass spectometry-based techniques. For the time being, the "gold standard" for the determination of methylation patterns remains bisulfite sequencing which allows a precise determination of each CpG site within a few hundred base pairs on individual alleles, but mass spectrometry techniques are catching up.

Each of these methods is useful for the purpose of cancer diagnosis in one or the other case. Principally, two different aims can be pursued by DNA methylation assays: cancer detection and cancer classification. Each aim implies different requirements, as discussed below, but both benefit from certain properties of DNA and DNA methylation. DNA is a more stable molecule than RNA and most proteins, facilitating sampling in a clinical routine setting. Similarly, DNA methylation patterns do not change rapidly in vivo (they usually require one or two complete cell cycles to be reset) and remain stable in isolated DNA (the 5-position of cytosine is among the least reactive ones, unless activated by methyltransferases). Perhaps most importantly, some sites in DNA are never methylated in normal cells, for example, many of those located in CpG-islands. Therefore, determination of hypermethylation, in particular, can often be performed against a true zero background. Hypomethylation detection is more tedious, since methylation of a single site may exceed $99 \%$ (ie, the site is methylated in 99 out of 100 alleles), but does not usually reach $100 \%$ (ie, a specific site remains unmethylated on an occasional allele). This yields a theoretical and practical limit, but combining several sites of this kind still can yield extraordinarily high specificity and sensitivity.

Nevertheless, as a rule, hypermethylation assays seem to constitute the method of choice for cancer detection. Because of the low background methylation in normal cells, the sensitivity of hypermethylation assays is usually not limited by the technique used, but by the biological properties of the cancer in question. Ideally, one or several genes would be consistently hypermethylated in a certain cancer type, but such cases are rare. Prostate cancer may be singular in that half a dozen genes including GSTP1 are coordinately hypermethylated at an early stage of its development in more than $80 \%$ of all cases. Thus, between $85 \%$ and $95 \%$ of all prostate cancers may be detectable by a small panel of hypermethylation assays. Use of several genes for detection is advisable not only because it increases the sensitivity of the assay, but also because it is more robust and discriminates better against methylation changes in preneoplastic or aging tissues. Unfortunately, in most other cancer types, no single gene is hypermethylated in such a large fraction of the cases and even combinations of methylation markers do not detect all. Some cancers, such as common type renal cell carcinoma, present very few methylation changes, especially when compared to aging tissues. The biological basis of these differences is obviously extremely interesting, but not understood. It has been postulated that some tumors exhibit a "methylator phenotype" which leads to hypermethylation as the preferred mode of tumor suppressor inactivation (compared to deletion and point mutation). Others have suggested that there is rather a gradual range of tumors with some exhibiting very few and others many methylation changes. At any rate, the prospects for the use of methylation assays for detection appear to vary considerably for different cancer types.

Another important consideration concerns the choice of samples for cancer detection. Investigating biopsies from a presumed cancerous tissue may be hampered by sampling error only. Similarly, detecting lung cancer in sputum or bladder cancer in urine by investigating the DNA of cells shed into the lumen is likely to be reliable. A different issue is detection of altered methylation in DNA from blood, a sort of assay usually performed with cellfree DNA present in plasma. This type of detection may be biased towards detection of advanced tumors in close contact with the vasculature and may in fact represent a sort of "molecular staging." With this consideration in mind, of course, detection of cells with aberrantly methylated DNA in lymph nodes or bone marrow may turn out to be highly useful in the clinic.

In a sense, this latter application transcends the border into cancer classification. In general, classification of cancers by DNA methylation analysis is applicable for several purposes, including the distinction between histological subtypes, between cancers of different stages (see above) or grades, between morphologically similar cancers with different prognoses, and between cancers responding differently to different treatments. It is commonly assumed that making such distinctions will require the analysis of multiple methylation sites in multiple genes. Most likely, assays for this purpose will comprise hypomethylation as well as hypermethylation analyses, because increasing hypomethylation is often a feature of more aggressive cancers. Moreover, as many sites will have to be sampled, techniques suitable for high-throughput or automation are expected to be employed. Obviously, cancer classification by methylation analysis will be a considerable more difficult task than cancer detection. One foreseeable complication, already experienced in expression profiling studies, is that the classifications of human cancers suggested by molecular markers may follow established division lines in some cases, but may well run contrary to them in others. This may be particularly so with methylation analyses because methylation changes drive cancer development to different degrees in individual cases of the same cancer type. For instance, individual colon cancers differ so substantially in the extent of methylation alterations that a subgroup with a "methylator phenotype" has been postulated.

Therefore, in the short run, the most likely application for classification based on methylation analysis might be the molecular distinction between histological types in the same organ (e.g., different lung or testicular cancers), because the differences in methylation are rather clear-cut and in accordance with established histological subtyping. A second application likely to enter clinical practice soon is prediction of chemotherapy response, since it is 
pursued actively and jointly by academic groups and industry. Specifically, hypermethylation of DNA repair, apoptosis, and checkpoint genes may presage the success of "classical" cytotoxic chemotherapy, but perhaps even the efficacy of "novel" targeted drugs in individual patients.

Finally, a prerequisite to make the promise of DNA methylation assays come true as they move from research labs into the clinic is improved standardization and quality control. A few examples may serve to indicate the scale of the problem. One report indicated a 100\% sensitivity and specificity for detection of renal cancers by methylation analysis from urine, whereas in others hypermethylation was only observed in a fraction of primary renal cancer tissues. Another paper reported 100\% hypermethylation of the p14 promoter in the CDKN2A gene in a cancer type where at least $20 \%$ of the cases carry homozygous deletions of the gene. Even the reported frequencies of a basically robust marker, like GSTP1 hypermethylation in prostate cancer, vary between $36 \%$ and $99 \%$ in different reports. Obviously, standardization of DNA methylation assays is anything but a trivial issue. Some discrepancies may result from technicalities or exaggerated claims, but the very properties of DNA methylation as a biological process may contribute. DNA methylation patterns are rarely $100 \%$ identical between cells of one type, even those of a clonal population, and they are dynamic. Thus, a densely hypermethylated CpG-island in a cancer cell may contain a few unmethylated CpG sites that vary between individual alleles. Moreover, hypermethylation is not dense in each gene affected in human cancers, so some of the variability found in the literature may derive from this fact. Moreover, DNA methylation patterns can change during the course of a cancer. The best documented case concerns the $\mathrm{CDH} 1$ gene encoding ECadherin. The gene is down-regulated and to varying extents hypermethylated when carcinoma cells undergo a cadherin isotype switch associated with an invasive phenotype. Conversely, however, expression of the protein is advantageous for metastases once they are established. Accordingly, methylation of the gene promoter may decrease again in metastases. Given this dynamic behavior, the irritating differences in the frequencies of $\mathrm{CDH} 1$ hypermethylation reported, for example, in prostate and bladder cancer become more understandable.

In conclusion, DNA methylation assays hold great promise for the better detection and classification of many human cancers, even if the limits of the approach are critically considered. Many techniques are applicable, depending on the purpose. Sensitivity, standardization, quality control, and automation are being developed and will facilitate translation into the clinic. Importantly, to make promise come true, the biological basis of DNA methylation alterations needs to be thoroughly understood for each human cancer type.
Wolfgang Schulz is Research Director at the Department of Urology, Heinrich Heine University, Duesseldorf, Germany. He studied biochemistry at the University of Tübingen and received his $\mathrm{PhD}$ there in 1985. To pursue cancer research, he underwent postdoctoral training at the Department of Pathology, University of Pittsburgh, $\mathrm{Pa}$, and at the Department of Clinical Genetics at the University of Ulm. In Duesseldorf, he first joined the Institute of Physiological Chemistry to study regulation of DNA methylation and human glutathione transferase genes. After moving to the Department of Urology, work in his research group has centered around DNA methylation alterations in urological cancers, addressing their causes, consequences, and their use for cancer diagnostics. Related projects deal with the mechanisms causing chromosomal instability in prostate and bladder cancer (which correlates with DNA hypomethylation) and the mechanisms driving aberrant proliferation of bladder cancer cells (to which DNA hypermethylation contributes). In line with his pursuit of "translational research," Professor Schulz is dedicated to interdisciplinary teaching of molecular medicine and molecular oncology. His textbook "Molecular Biology of Human Cancer: An Advanced Student's Textbook” has recently appeared. 


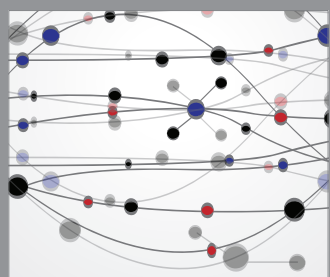

The Scientific World Journal
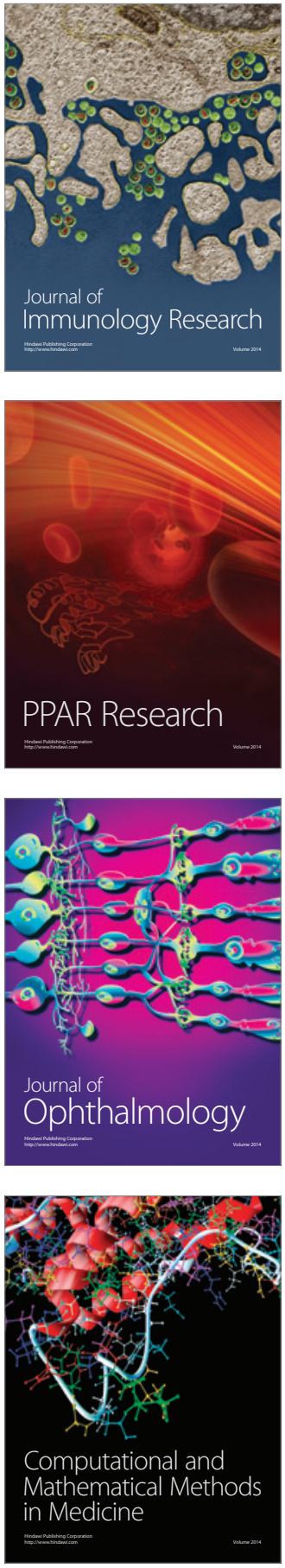

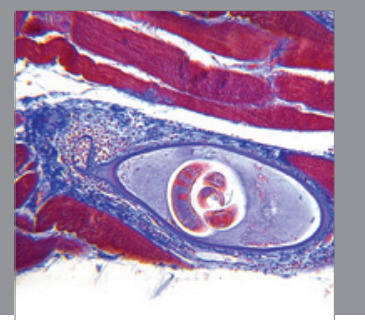

Gastroenterology

Research and Practice
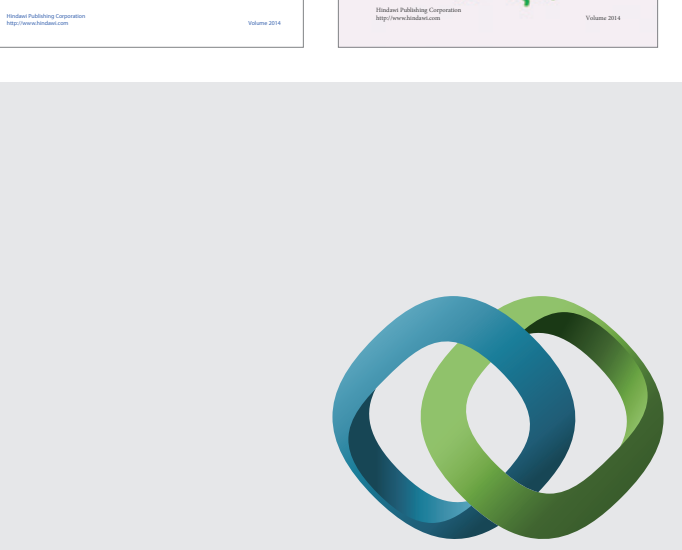

\section{Hindawi}

Submit your manuscripts at

http://www.hindawi.com
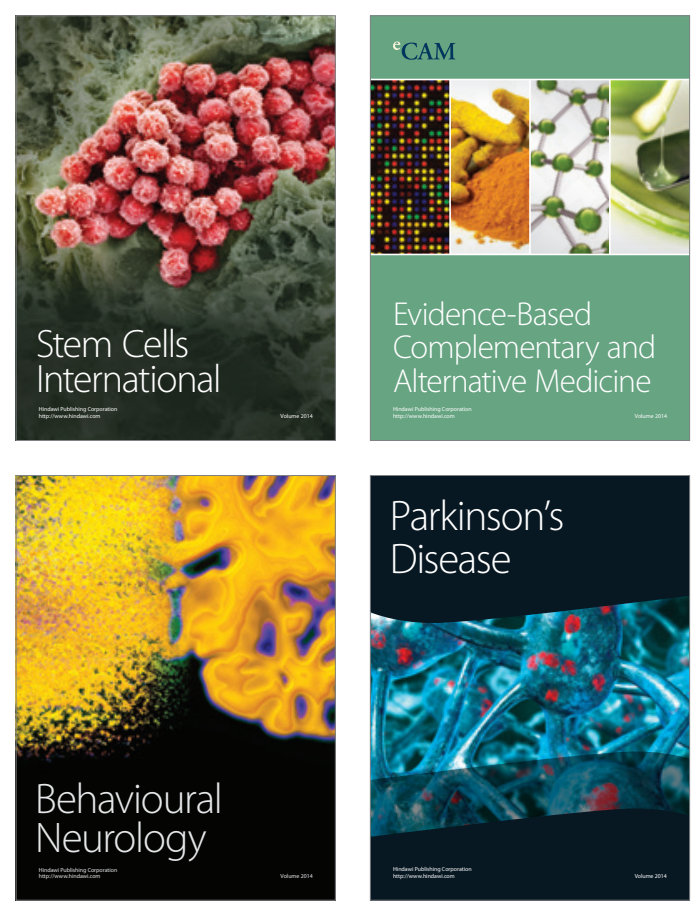

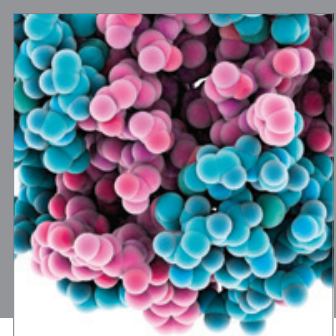

Journal of
Diabetes Research

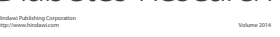

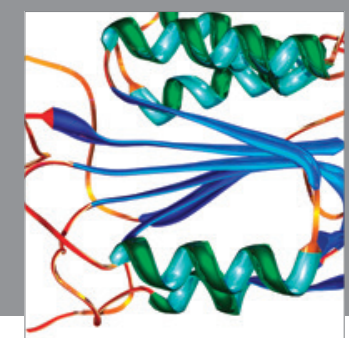

Disease Markers
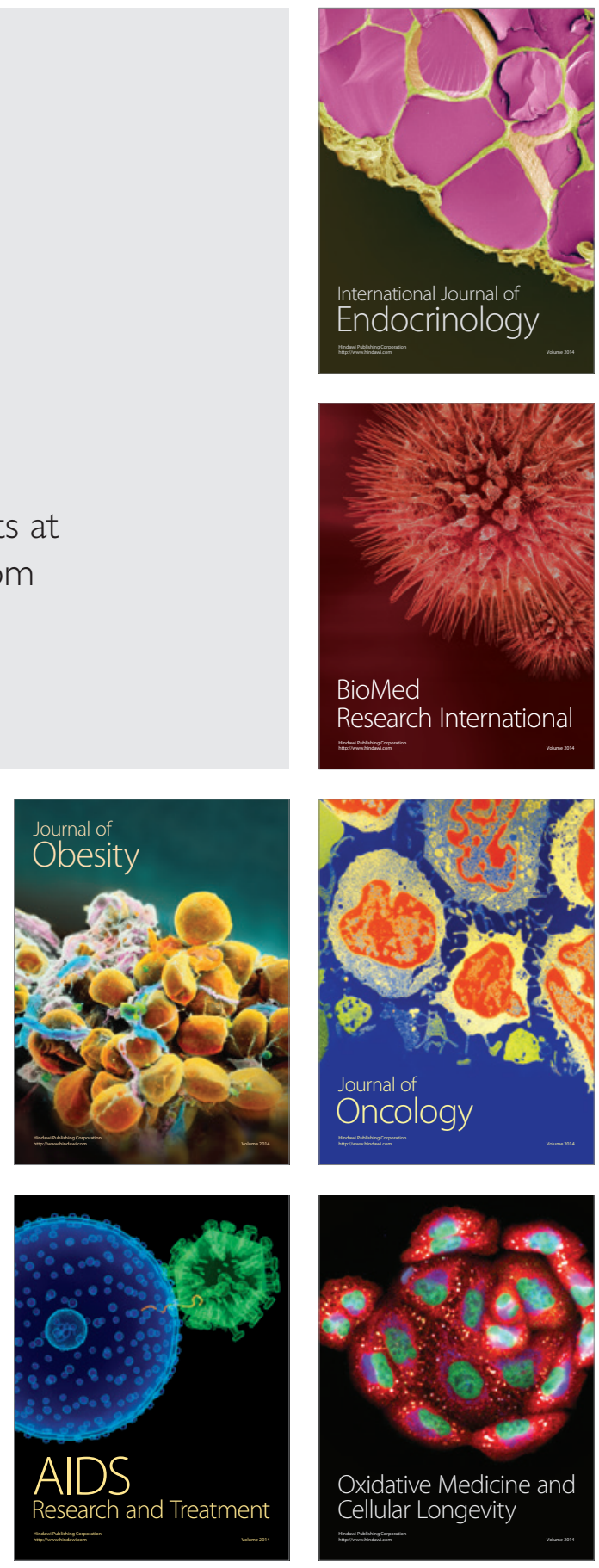